\section{REVISTA}

Actualidades Investigativas en Educación http://revista.inie.ucr.ac.cr/

ISSN 1409-4703

\title{
EDUCACIÓN AMBIENTAL PARA LA SUSTENTABILIDAD EN LA
} EDUCACIÓN SECUNDARIA

ENVIRONMENTAL EDUCATION FOR SUSTAINABILITY IN SECONDARY EDUCATION

\section{Volumen 15, Número 3}

Setiembre - Diciembre

pp.1-21

Este número se publicó el $1^{\circ}$ de setiembre de 2015

DOI: http://dx.doi.org/10.15517/aie.v15i3.20929

\author{
Raúl Calixto Flores
}

Revista indizada en REDALYC, SCIELO

Revista distribuida en las bases de datos:

CATÁLOGO DE LATINDEX, IRESIE, CLASE, DIALNET, DOAJ, E-REVIST@S, SHERPA/ROMEO, QUALIS, MIAR

Revista registrada en los directorios:

ULRICH'S, REDIE, RINACE, OEI, MAESTROTECA, PREAL, CLACSO 


\title{
EDUCACIÓN AMBIENTAL PARA LA SUSTENTABILIDAD EN LA EDUCACIÓN SECUNDARIA \\ ENVIRONMENTAL EDUCATION FOR SUSTAINABILITY IN SECONDARY EDUCATION
}

Raúl Calixto Flores ${ }^{1}$

\begin{abstract}
Resumen. En este ensayo se analizan los programas de estudio del nivel medio básico de México en el campo de formación, exploración y comprensión del mundo natural y social, relacionado con la educación ambiental para la sustentabilidad y las implicaciones que se pueden derivar para la docencia (estos programas tienen un carácter nacional). En los programas de estudio analizados se encuentran elementos de la sustentabilidad débil, fuerte y súper fuerte. Estas categorías son propuestas por el especialista Eduardo Gudynas para diferenciar las distintas nociones de la sustentabilidad. En los resultados del análisis se observa el predominio de la sustentabilidad débil en los programas de estudio. La sustentabilidad débil se asocia más con las directrices del desarrollo sustentable que con las propuestas de la educación ambiental, por cuanto esta última propone aprovechar los recursos no sólo económicos sino también naturales con la intención de producir lo que la sociedad demanda. Esta visión ambiental, resalta la idea de cuidar los ecosistemas mediante la administración eficiente de los recursos. Como aporte del análisis realizado a los programas de estudio del nivel medio básico se destaca la importancia de incluir temas sociales y culturales en la docencia de la educación ambiental para la sustentabilidad. Esta perspectiva en la educación ambiental contribuye a la formación de una actitud crítica hacia los problemas ambientales, develando contradicciones sociales y construyendo alternativas comprometidas con el bien común.
\end{abstract}

Palabras clave: EDUCACIÓN AMBIENTAL, EDUCACIÓN SECUNDARIA, CURRICULUM, SUSTENTABILIDAD, DOCENTES, MÉXICO

Abstract: In this essay is analyzed the curriculum of secondary level of Mexico in the field of training, exploration and understanding of the natural and social world, related to environmental education for sustainability and the implications that can be derived for teaching. These programs have a national character. In the curricula are analyzed elements of weak, strong and super-strong sustainability. Categories proposed by Gudynas (2009) in order to distinguish different notions of sustainability. In the analysis results the prevalence of weak sustainability in the curriculum is observed. Weak sustainability is more associated with the guidelines of sustainable development that in environmental education proposals, in the way that proposes use resources both as the economic as the natural with the intention of producing the products that society demands. It also highlights the idea of ecosystem stewardship through the efficient management of resources. Among the conclusions it emphasizes the importance of including social and cultural issues in the teaching of environmental education for sustainability. This perspective on environmental education contributes to the formation of a critical attitude towards environmental problems, revealing social contradictions and building alternatives committed to the common good.

Keywords: ENVIRONMENTAL EDUCATION, HIGH SCHOOL EDUCATION, EDUCATIONAL PROGRAM, SUSTAINABILITY, TEACHERS, MEXICO

\footnotetext{
Profesor-Investigador de la Universidad Pedagógica Nacional, México. Área académica: Diversidad e Interculturalidad.
}

Dirección electrónica: raul 2020@outlook.com

Ensayo recibido: 3 de octubre, 2014

Enviado a corrección: 16 de marzo, 2015

Aprobado: 13 de julio, 2015 


\section{Introducción}

La crisis ambiental ha llegado a un grado tan alarmante que ahora se hace necesario, por medio de la educación, tomar conciencia de la importancia de cambiar las formas de producción y bienestar social, como también del respeto a la diversidad cultural y condiciones que posibilitan la existencia de vida en el planeta.

En el este contexto se hace evidente la importancia de la educación ambiental, como una de las alternativas para el reconocimiento del valor de la conservación de las condiciones naturales del planeta. En los últimos años, la educación ambiental ha sido vinculada al desarrollo sustentable.

A partir de la publicación del Informe Nuestro Futuro Común en 1987, también conocido como Reporte Brundtland se difundió internacionalmente el concepto de desarrollo sustentable, como aquél que satisface las necesidades del presente, sin comprometer la capacidad de que las futuras generaciones puedan satisfacer las propias. Entre otras cuestiones se plantea que el futuro del planeta depende en gran medida de acciones políticas orientadas a un manejo adecuado de los recursos naturales.

Desde entonces y a lo largo de los últimos años, el concepto de desarrollo sustentable se encuentra en los más variados ámbitos de la vida pública, entre ellos y, de manera especial, el educativo.

En diciembre de 2002 la Asamblea General de Naciones Unidas proclamó el periodo 2005-2014, como "La Década para la Educación por el Desarrollo Sustentable", con el propósito fundamental de preparar e involucrar a los individuos y grupos sociales en la reflexión sobre nuestro modo de vida, en la toma de decisiones informadas y en el establecimiento de vías para avanzar hacia un mundo mejor. Esta propuesta pretende articular esfuerzos con otras iniciativas, como el Plan de Acción de Dakar sobre Educación para Todos, los Objetivos de Desarrollo del Milenio, la Década de Alfabetización, la Educación para la Prevención del VIH-SIDA, y con otros proyectos educacionales en curso, como el Proyecto Regional de Educación para América Latina y el Caribe (UNESCO), Centro Carta de la Tierra de Educación para el desarrollo sostenible (2007), entre otros La Década por la Educación para el Desarrollo Sustentable propone, entre otras cuestiones, que los profesores (desde la escuela primaria a la universidad) atiendan la problemática ambiental fomentando en sus estudiantes actitudes y comportamientos favorables para el logro de un desarrollo sustentable. 
El desarrollo sustentable ha sido promovido por las instancias internacionales, en particular por la Comisión de Desarrollo Sustentable (CDS), ligada al Consejo Económico y Social de la Organización de las Naciones Unidas. Este polisémico concepto tiene implicaciones directas en la política económica de las naciones y apela a una articulación funcional entre la economía, la ecología y la sociedad, que ha sido ampliamente aceptado, aunque no apropiadamente instrumentado. En México, la Secretaría de Educación Pública (SEP) y la Secretaria del Medio Ambiente y Recursos Naturales (SEMARNAT) han dado lugar al enfoque de la educación ambiental para la sustentabilidad. Enfoque que está presente en los actuales programas de estudio del nivel medio básico.

En este ensayo se analizan los programas de estudio del nivel medio básico de México en el campo de formación exploración y comprensión del mundo natural y social, relacionado con la educación ambiental para la sustentabilidad y con los resultados de este análisis se observan las implicaciones que tienen los contenidos de estos programas para la docencia.

\section{Análisis de los programas}

En México, como en otros países latinoamericanos la propuesta de la UNESCO de educación para el desarrollo sustentable, da lugar a la orientación hacia la sustentabilidad en la educación ambiental.

En América Latina la orientación que toma la educación ambiental para la sustentabilidad es, en general, muy crítica con los modelos de desarrollo dominantes, marcadamente militante y comprometida con los conflictos y problemas ambientales de cada contexto singular. Las problemáticas ambientales son vistas más que exclusivamente ecológicas derivadas del funcionamiento de la propia naturaleza o de los procesos de industrialización y acción tecnológica, como cuestiones de alto nivel de complejidad que conlleva asociados de manera natural consecuencias sociales (graves, en muchos casos). (Gutiérrez y Priotto, 2008, p. 537)

Al prescindir del término de desarrollo y utilizar solo el de sustentabilidad se da un giro de 180 grados a la propuesta de la UNESCO, ya que se centra la atención en la conformación de una nueva visión del mundo, en una nueva forma de observar las relaciones Estado-Sociedad, involucrando a la ciudadanía en las transformaciones institucionales y culturales. 
Aún falta mucho por hacer respecto a la educación ambiental en el nivel medio básico en México, entre otras cuestiones, los planes y los programas requieren de impregnarse de la dimensión ambiental que replantee la visión dominante de la relación sociedad-naturaleza. Así como de un enfoque sustentable que retome como elementos relevantes el origen de las desigualdades sociales y de los problemas ambientales.

La educación ambiental representa una solución o alternativa básica a la problemática socio-ambiental. Para ello, se deben promover nuevos estilos pedagógicos que conduzcan a la revalorización del individuo como ser social, cultural, independiente y capaz de potenciar cambios en la sociedad postmodernista. (Martínez, 2007, p. 10)

La educación ambiental en el nivel medio básico o educación secundaria representa una oportunidad para consolidar los valores ambientales de los adolescentes en favor de un futuro sustentable.

En este ensayo se analizan los actuales programas de estudio en el nivel medio básico, relacionados con la educación ambiental para la sustentabilidad, se identifican algunas de sus implicaciones para la docencia.

En el análisis de contenidos se asume una perspectiva sistémica, por lo tanto el análisis no es secuencial, es cíclico, por lo que permite la comparación entre los elementos que lo conforman.

Para Krippendorff (1990), el análisis de contenido permite formular, a partir de ciertos datos, inferencias reproducibles y válidas que puedan aplicarse a un contexto, lo cual sitúa al investigador con respecto a la realidad en una triple perspectiva: los datos tal y como se comunican al analista, el contexto de los datos y la forma en que el conocimiento del analista obliga a dividir la realidad. Un primer nivel de análisis es el descriptivo, preservando su naturaleza textual; Piñuel y Gaitán (1995) consideran que el análisis de contenido proporciona las herramientas para la des-ocultación o re-velación de la expresión.

En este tipo de análisis se supone que el contenido está encerrado en un "continente" (documento físico) y analizándolo se puede develar su contenido (significado o sentido); al analizar con detalle el contenido de cualquier material de comunicación humana, se determinan los objetivos que se pretenden alcanzar; se define el universo que se pretende estudiar; se eligen los documentos; se selecciona el cuerpo de unidades de contenido o de análisis y; finalmente se define el proceso de análisis con la determinación de las categorías. El análisis de contenido es una técnica objetiva y sistemática muy útil para identificar los 
aspectos relevantes de los programas de las asignaturas de educación secundaria.

El segundo nivel de análisis es interpretativo, en él "Se relacionan los datos obtenidos con otros trabajos o estudios y con marcos analíticos más generales, dentro de los que cobran sentido los datos estudiados..." (Porta y Silva, 2003, p.16). Este análisis posibilitó identificar la implicaciones para la docencia del enfoque de la sustentabilidad.

El corpus de análisis está integrado por el plan de estudios del nivel medio básico y las asignaturas del campo, exploración y comprensión del mundo natural y social. Se trabajaron tres categorías de la sustentabilidad: débil, fuerte y súper-fuerte derivadas de la propuesta de Gudynas (2009).

Gudynas (2009), denomina sustentabilidad débil, a la que incluye soluciones técnicas para los problemas ambientales; sustentabilidad fuerte a la que utiliza, principalmente, soluciones técnicas y de valoración económica, pero también considera la necesidad de otros componentes para lograr la sustentabilidad y; la súper-fuerte, propone cambios mayores, que incluye el desarrollo de valores ecológicos, estéticos, religiosos y culturales. Los principales atributos de cada categoría se presentan en la tabla 1.

\section{Tabla 1. Atributos de la sustentabilidad}

\begin{tabular}{|l|l|}
\hline \multicolumn{1}{|c|}{ Categoría } & \multicolumn{1}{|c|}{ Atributos } \\
\hline $\begin{array}{l}\text { Sustentabilidad } \\
\text { Débil }\end{array}$ & $\begin{array}{l}\text { Acepta cambios en los procesos productivos actuales para reducir el } \\
\text { impacto ambiental; la conservación es necesaria para el crecimiento } \\
\text { económico. Se enfatizan las soluciones técnicas a los problemas } \\
\text { ambientales. }\end{array}$ \\
\hline $\begin{array}{l}\text { Sustentabilidad } \\
\text { fuerte }\end{array}$ & $\begin{array}{l}\text { Acepta la necesidad de cambios radicales en el modelo económico y en la } \\
\text { sociedad para facilitar un reparto equitativo de los costos y beneficios } \\
\text { ambientales (equidad intra e inter-generacional). Subraya la necesidad de } \\
\text { asegurar la supervivencia de especies y la protección de ambientes } \\
\text { críticos, más allá de su posible uso económico. }\end{array}$ \\
\hline $\begin{array}{l}\text { Sustentabilidad } \\
\text { súper-fuerte }\end{array}$ & $\begin{array}{l}\text { Acepta que el ambiente sea valorado de muy diferentes maneras, además } \\
\text { de la económica: también existen valores culturales, ecológicos, religiosos } \\
\text { o estéticos, que son tanto o más importantes; el concepto de "Patrimonio } \\
\text { Natural", es entendido como un acervo que se recibe en herencia de } \\
\text { nuestros antecesores y que debe ser mantenido, legado a las } \\
\text { generaciones futuras, y no necesariamente vendible o comprable en el } \\
\text { mercado. }\end{array}$ \\
\hline
\end{tabular}

Fuente: Eduardo Gudynas, 2009.

La sustentabilidad débil se asocia más a las directrices del desarrollo sustentable, y éstas a su vez se relacionan con la educación ambiental conservacionista, en el sentido que propone aprovechar los recursos, no sólo económicos sino también los naturales, con la 
intención de producir los satisfactores que la sociedad demanda. También, resalta la idea de cuidar los ecosistemas mediante la administración eficiente de los recursos.

La sustentabilidad fuerte se asocia con la educación ambiental para la sustentabilidad ya que incorpora en sus acciones condiciones para que la vida perdure, se consideran aspectos ambientales, sociales y políticos. Comprende contenidos referidos al equilibrio real entre las especies vivas y la utilización de los recursos, se considera la satisfacción de las necesidades futuras de los otros con miras al desarrollo económico y se enfatiza en el cuidado el medio ambiente al llevar a cabo cualquier actividad.

La sustentabilidad súper-fuerte se vincula con la educación popular ambiental, considera la educación como una propuesta transformadora que aporta elementos para un cambio de creencias, actitudes, hábitos, conocimientos y comportamientos que impacten de una manera positiva en el ambiente y con ello favorecer una relación más amigable del ser humano con el ambiente.

\section{Los elementos que predominan en los programas de estudio}

La educación ambiental incluye contenidos transversales que se abordan, con diferentes énfasis, en varias asignaturas. En el "acuerdo 384" de la SEP que sustenta el plan de estudios del nivel medio básico (2011a), se señala que estos contenidos están conformados por temas que contribuyen a propiciar una formación crítica para que los estudiantes reconozcan los compromisos y las responsabilidades que les atañen con su persona y la sociedad en que viven. Estos contenidos se encuentran organizados en tres ejes o campos de estudio:

- Educación ambiental.

- La formación en valores.

- Educación sexual y equidad de género.

En cuanto al primer eje, la educación ambiental, se proponen entre otros aspectos, los que abordan los problemas ambientales en el plano local, nacional y mundial. En cuanto al segundo eje, se propone construir un marco general de valores, entre los que se incluyen, la libertad, la igualdad, la solidaridad, la justicia, el aprecio y el respeto a la vida, a la diversidad cultural y a la dignidad de las personas. $Y$ en el tercer eje, educación sexual y género, se propone un conocimiento amplio de la sexualidad, en el que estén presentes, entre otras dimensiones, las de afecto, género, reproducción y disfrute; las actitudes de aprecio y 
respeto por uno mismo y por los demás; y la información confiable sobre diversos aspectos de la sexualidad como los embarazos tempranos.

En los programas se proponen un perfil de egreso en el estudiante como resultado del proceso de formación a lo largo de la Educación Básica; perfil con diversos rasgos, entre éstos se encuentran el de formar estudiantes en el cuidado de la salud y del ambiente como condiciones para favorecer un estilo de vida activo y saludable. Para lograr este perfil de egreso se proponen los siguientes campos de formación en la educación básica:

- Lenguaje y comunicación.

- Pensamiento matemático.

- Exploración y comprensión del mundo natural y social.

- Desarrollo personal y para la convivencia.

En este ensayo solo se aborda el campo de formación: Exploración y comprensión del mundo natural y social, se integran diversos enfoques disciplinares relacionados con aspectos biológicos, históricos, sociales, políticos, económicos, culturales, geográficos y científicos. En este campo de formación se pretende entre otras cuestiones que los estudiantes desarrollen la perspectiva de explorar y entender el entorno mediante el acercamiento sistemático y gradual a los procesos sociales y fenómenos naturales. Entre los que se encuentran los temas ambientales a nivel regional, nacional o mundial, conforme se avanza en los grados escolares.

En el mapa curricular de la educación básica 2011, se observa la integración de las asignaturas en cada uno de los bloques de formación. Los jóvenes de las escuelas secundarias se encuentran en el último ciclo de la educación básica, por lo general en las escuelas primarias han estudiado, leído o escuchado contenidos referidos al cuidado del medio ambiente. Ya que en los planes de estudio y en los programas los temas ambientales ocupan un espacio relevante.

Campo de formación: Exploración y comprensión del mundo natural y social en el mapa curricular de la educación básica (Tabla 2): 
Tabla 2. Mapa Curricular de la Educación Básica

\begin{tabular}{|c|c|c|c|c|c|c|c|c|c|c|c|c|}
\hline \multirow{3}{*}{$\begin{array}{c}\text { Campo de } \\
\text { Formación } \\
\text { para la } \\
\text { Educación } \\
\text { Básica }\end{array}$} & \multicolumn{3}{|c|}{$\begin{array}{l}\text { Primer } \\
\text { período } \\
\text { escolar }\end{array}$} & \multicolumn{3}{|c|}{$\begin{array}{l}\text { Segundo período } \\
\text { escolar }\end{array}$} & \multicolumn{3}{|c|}{$\begin{array}{l}\text { Tercer } \\
\text { período } \\
\text { escolar }\end{array}$} & \multicolumn{3}{|c|}{ Cuarto período escolar } \\
\hline & \multicolumn{3}{|c|}{ Preescolar } & \multicolumn{6}{|c|}{ Primaria } & \multicolumn{3}{|l|}{ Secundaria } \\
\hline & $1 \stackrel{0}{ }$ & $2^{\circ}$ & $3^{\circ}$ & 10 & $2^{\circ}$ & $3^{\circ}$ & $4 \div$ & $5^{\circ}$ & 6 & 10. & $2^{\circ}$ & $3^{\circ}$ \\
\hline \multirow{4}{*}{$\begin{array}{l}\text { Exploración y } \\
\text { Comprensión } \\
\text { del Mundo } \\
\text { Natural y } \\
\text { Social }\end{array}$} & \multirow{2}{*}{\multicolumn{3}{|c|}{$\begin{array}{l}\text { Exploración y } \\
\text { conocimiento } \\
\text { del mundo }\end{array}$}} & \multirow{4}{*}{\multicolumn{2}{|c|}{$\begin{array}{l}\text { Exploración } \\
\text { de la } \\
\text { naturaleza y } \\
\text { la sociedad }\end{array}$}} & \multirow{4}{*}{$\begin{array}{l}\text { La } \\
\text { entidad } \\
\text { donde } \\
\text { vivo }\end{array}$} & \multicolumn{3}{|c|}{$\begin{array}{l}\text { Ciencias } \\
\text { Naturales }\end{array}$} & $\begin{array}{l}\text { Ciencias I } \\
\text { (énfasis } \\
\text { Biología) }\end{array}$ & $\begin{array}{l}\text { Ciencias } \\
\text { II } \\
\text { (énfasis } \\
\text { Física) }\end{array}$ & $\begin{array}{l}\text { Ciencias } \\
\text { III } \\
\text { (énfasis } \\
\text { Química) }\end{array}$ \\
\hline & & & & & & & \multirow{2}{*}{\multicolumn{3}{|c|}{ Geografía }} & \multicolumn{3}{|c|}{ Tecnología I, II y III } \\
\hline & \multirow{2}{*}{\multicolumn{3}{|c|}{$\begin{array}{l}\text { Desarrollo } \\
\text { físico y salud }\end{array}$}} & & & & & & & $\begin{array}{l}\text { Geografía } \\
\text { de México y } \\
\text { del Mundo }\end{array}$ & Historia & \\
\hline & & & & & & & His & & & $\begin{array}{l}\text { Asignatura } \\
\text { Estatal }\end{array}$ & & \\
\hline
\end{tabular}

Fuente: SEP, 2011b.

En educación secundaria, con las asignaturas de Ciencias, Geografía de México y del Mundo, Historia, Tecnología y la Asignatura Estatal se culmina el campo de formación exploración y comprensión del mundo natural y social. En el Plan de Estudios 2011, se describen cada una de las asignaturas de educación secundaria. En la tabla 3 se muestran algunos de los principales aspectos de las asignaturas anteriormente referidas.

Tabla 3. Aspectos de las asignaturas que conforman el campo de formación Exploración y comprensión del mundo natural y social

\begin{tabular}{|l|l|}
\hline \multicolumn{1}{|c|}{ Asignatura } & \multicolumn{1}{c|}{ Aspectos } \\
\hline Ciencias & $\begin{array}{l}\text { Los estudiantes se aproximan al estudio de los fenómenos de la naturaleza } \\
\text { y de su vida personal de manera gradual y con explicaciones metódicas y } \\
\text { complejas, y buscan construir habilidades y actitudes positivas asociadas a } \\
\text { la ciencia. Así como favorecer la toma de decisiones responsables e } \\
\text { informadas a favor de la salud y el ambiente }\end{array}$ \\
\hline $\begin{array}{l}\text { Geografía de México } \\
\text { y del Mundo }\end{array}$ & $\begin{array}{l}\text { componamnos se asumen como parte del espacio geográfico, valoren los } \\
\text { dinámica de la población mediante los componentes sociales y culturales, } \\
\text { así como la desigualdad socioeconómica para fortalecer su participación de } \\
\text { manera informada, reflexiva y crítica ante los problemas sociales, el } \\
\text { cuidado del ambiente, la vulnerabilidad de la población y la calidad de vida } \\
\text { en las escalas nacional y mundial. }\end{array}$ \\
\hline Historia & $\begin{array}{l}\text { Los estudiantes comprenden el mundo donde viven para ubicar y darle } \\
\text { importancia a los acontecimientos de la vida diaria, y usar críticamente la } \\
\text { información para convivir con plena conciencia ciudadana. }\end{array}$ \\
\hline Tecnología & $\begin{array}{l}\text { A los estudiantes se les orienta al estudio de la técnica y sus procesos de } \\
\text { cambio, considerando sus implicaciones en la sociedad y en la naturaleza; } \\
\text { entre otras cuestiones se busca que sepan tomar decisiones de manera } \\
\text { responsable en el uso y la creación de productos y procesos técnicos. }\end{array}$ \\
\hline
\end{tabular}




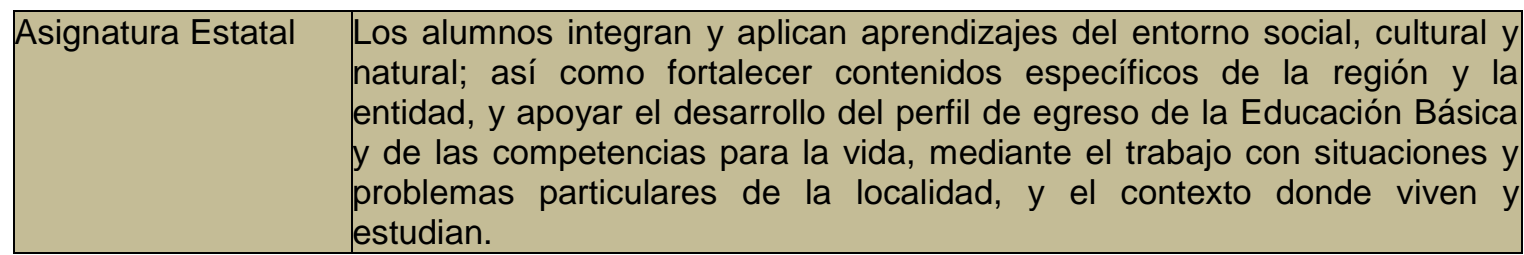

Fuente: SEP, 2011a.

La asignatura Estatal se cursa en el primer grado de la educación secundaria; la SEP establece los lineamientos nacionales donde se especifican los campos temáticos de esta asignatura: 1) La historia, la geografía y/o el patrimonio cultural de la entidad;2) Educación ambiental para la sustentabilidad; 3) Estrategias para que los alumnos enfrenten y superen problemas y situaciones de riesgo y, 4) Lengua y cultura indígena.

La educación ambiental en el nivel medio básico se orienta hacia la sustentabilidad, se propone fomentar la participación reflexiva del alumno ante los problemas ambientales, los cuales constituyen una realidad compleja que necesariamente debe ser abordada desde todas sus dimensiones. Entre los principios pedagógicos de la educación básica, señalado en el Plan de estudios 2011, se hace referencia a la necesidad de incorporar temas de relevancia social para que los estudiantes aprendan actuar con responsabilidad ante el medio natural y social, y se refieren a la educación ambiental para la sustentabilidad, entre otros más.

De acuerdo al enfoque de las asignaturas referidas al campo de formación exploración y comprensión del mundo natural y social, se espera que los estudiantes de educación secundaria, además de conocer diversos temas relacionados con el medio ambiente, desarrollen las competencias para la vida que les permitan obtener la sensibilidad hacia los acontecimientos y problemas ambientales de nuestro tiempo y la habilidad para identificar y descubrir problemas ambientales, como también las habilidades para idear métodos y medios para contribuir a resolver o reducir esos problemas. Así como fomentar la conciencia de los problemas ambientales, y los valores y actitudes, para la protección y conservación del medio ambiente.

Entre los rasgos del perfil de egreso de los estudiantes en este nivel educativo:

Emplea los conocimientos adquiridos con el fin de interpretar y explicar procesos sociales, económicos, culturales y naturales, así como para tomar decisiones y actuar, individual o colectivamente, en aras de promover la salud y el cuidado ambiental, como formas para mejorar la calidad de vida. (SEP a, 2006, p. 3) 
Las asignaturas estatales. La Secretaría de Educación Pública, a través de la Subsecretaría de Educación Básica, la Dirección General de Desarrollo Curricular y la Dirección de Enlace y Vinculación, conforme al acuerdo secretarial número 384 y los lineamientos nacionales 2009 para el diseño y elaboración de los programas de la asignatura estatal, establecen la inclusión en los programas de las asignaturas estatales de alguno de los siguientes campos temáticos:

Campo temático 1. La historia, la geografía y/o patrimonio cultural y natural de la entidad.

Campo temático 2. El estudio de los temas que abordan en más de una asignatura: educación ambiental, formación en valores, y educación sexual y equidad de género.

Campo temático 3. Estrategias para que los alumnos enfrenten y superen problemas y situaciones de riesgo.

Campo temático 4. Estrategias que fortalecen las habilidades para el estudio y el aprendizaje.

Campo temático 5.Lengua y cultura indígena.

El diseño de cada asignatura estatal corresponde a la autoridad educativa estatal y los equipos académicos designados para tal fin, quienes en coordinación con los directivos y profesores de educación secundaria, eligen el tema y los contenidos del programa, para ello se deben de fundamentar en la información que se disponga de los problemas y necesidades locales. Los programas aprobados referidos a la educación ambiental en la asignatura estatal en el ciclo escolar 2012-2013 se observan en la tabla 4: 
Tabla 4. Asignatura estatal (2012-2013)

\begin{tabular}{|c|c|}
\hline Estado & Programa \\
\hline Aguascalientes & Educación ambiental para la sustentabilidad en Aguascalientes \\
\hline Baja California Sur & Jóvenes sudcalifornianos por una cultura de la sustentabilidad ambiental \\
\hline Campeche & Educación ambiental en el estado de Campeche \\
\hline Chiapas & Educación ambiental para la sustentabilidad en Chiapas \\
\hline Distrito Federal & Educación ambiental para el desarrollo sustentable en el Distrito Federal \\
\hline Durango & Cultura del agua para la sustentabilidad en el estado de Durango \\
\hline Guerrero & Cultura ambiental: Reto de los Guerrerenses \\
\hline Hidalgo & Educación ambiental para la sustentabilidad en Hidalgo \\
\hline Jalisco & Educación ambiental, adolescentes y sustentabilidad en Jalisco \\
\hline Estado de México & Educación ambiental para la sustentabilidad del Estado de México \\
\hline Michoacán & Educación ambiental para la sustentabilidad en Michoacán \\
\hline Morelos & Hacia una cultura ambiental en Morelos \\
\hline Querétaro & Cultura ambiental en Querétaro \\
\hline Quintana Roo & Educación y cultura ambiental para la sustentabilidad en Quintana Roo \\
\hline Tabasco & Educación ambiental para la sustentabilidad en Tabasco \\
\hline Tamaulipas & Educación ambiental para la sustentabilidad en Tamaulipas \\
\hline Tlaxcala & Educación ambiental para el desarrollo sustentable en Tlaxcala \\
\hline Veracruz & Educación para la sustentabilidad en Veracruz \\
\hline Zacatecas & Educación ambiental para la sustentabilidad en Zacatecas \\
\hline
\end{tabular}

Fuente: SEP, 2013a.

En estos programas se enuncia el enfoque de la educación ambiental para la sustentabilidad, en él se orienta hacia la formación de competencias referidas a la comprensión de la relación entre la sociedad y la naturaleza para favorecer el desarrollo sustentable y la valoración de la diversidad natural de la entidad.

Los programas tienen una estructura básica que comprende: presentación, introducción, enfoque didáctico, formación del alumno, competencias a desarrollar, papel del docente, modalidades de trabajo, recursos didácticos, organización de los aprendizajes, ejes formativos, descripción de los bloques de estudio, estructura de los bloques de estudio, bloques de estudio y fuentes bibliográficas y de consulta. Las estrategias didácticas que 
predominan son la de trabajo por proyectos y el estudio de casos.

El trabajo por proyectos. Esta estrategia implica el trabajo colaborativo de los estudiantes para analizar problemas ambientales (locales, regionales o estatales) y proponer acciones concretas; favorece las habilidades de investigación para obtener resultados propios y promueve la integración de los conocimientos de las distintas asignaturas para el desarrollo de las competencias formativas. El estudio de casos. En esta estrategia, se seleccionan situaciones reales, generalmente los problemas ambientales, de su contexto inmediato, para que los estudiantes apliquen los conocimientos aprendidos en el análisis de sus distintos componentes; como también los efectos y posibles acciones que mitiguen, prevengan o resuelvan el problema ambiental. A pesar de que comparten muchos elementos, cada programa estatal es diferente, ya que se enfoca a las cuestiones ambientales de cada Estado. En la tabla 5 se incluyen los nombres de los cinco bloques que aborda cada programa.

Tabla 5. Bloques de los programas

\begin{tabular}{|c|c|}
\hline Entidad & Programa \\
\hline \begin{tabular}{|l|} 
Aguas- \\
calientes
\end{tabular} & $\begin{array}{l}\text { 1. Aguascalientes y desarrollo sustentable: } 2 \text {. Riqueza natural y cultural del estado } \\
\text { de Aguascalientes; } 3 \text {. Cultura del agua en Aguascalientes; } 4 \text {. El binomio aire-suelo; } \\
\text { 5. Proyectos ambientales y sustentabilidad en Aguascalientes. }\end{array}$ \\
\hline $\begin{array}{l}\text { aja } \\
\text { aliforni }\end{array}$ & $\begin{array}{l}\text { 1. El ser humano y su entorno; } 2 \text {. Globalización; } 3 \text {. Nuestras riquezas naturales: } \\
\text { lagua, oasis y área naturales protegidas; } 4 \text {. Residuos sólidos; } 5 \text {. Sustentabilidad. }\end{array}$ \\
\hline Campeche & $\begin{array}{l}\text { 1. El entorno y su riqueza natural; 2. El agua como líquido vital: } 3 \text {. Consumo } \\
\text { responsable; } 4 \text {. Cultura ambiental; } 5 \text {. Desarrollo sustentable. }\end{array}$ \\
\hline Chiapas & $\begin{array}{l}\text { 1. Los adolescentes y su relación con el ambiente; } 2 \text {. La riqueza natural de Chiapas } \\
\text { y el desarrollo sustentable; } 3 \text {. El consumo sustentable del agua en Chiapas; } 4 \text {. El } \\
\text { cambio climático y otros problemas ambientales en Chiapas; } 5 \text {. Los adolescentes y } \\
\text { los estilos de vida sustentables }\end{array}$ \\
\hline Distrito & 1. El adolescente y su contexto natural; 2. Los estilos de vida en el Distrito Federal \\
\hline Feder & $\begin{array}{l}\text { y su relación con el ambiente; } 3 \text {. Riesgos ambientales y situaciones de } \\
\text { vulnerabilidad; } 4 \text {. Ciudadanía, cultura y valores ambientales. Un estilo de vida en } \\
\text { adolescentes; } 5 \text {. Desarrollo sustentable en el Distrito federal como un estilo de } \\
\text { vida. }\end{array}$ \\
\hline Durango & $\begin{array}{l}\text { El sistema natural del que forma parte; } 2 \text {. El sistema social en el uso del agua; } 3 \text {. } \\
\text { La relación naturaleza-sociedad; } 4 \text {. Acciones para la sustentabilidad del agua en mi } \\
\text { comunidad; } 5 \text {. Acciones para la sustentabilidad del agua en mi escuela y mi hogar. }\end{array}$ \\
\hline Estado & de1. Mi ambiente; 2. Deterioro ambiental en mi comunidad, municipio y Estado; 3. \\
\hline México & $\begin{array}{l}\text { Calidad de vida, deterioro ambiental y sustentabilidad en mi comunidad, municipio } \\
\text { y Estado; } 4 \text {. Consumo sustentable, estilos de vida y desarrollo sustentable; } 5 . \\
\text { Participación ciudadana y desarrollo sustentable en mi comunidad, municipio y } \\
\text { Estado. }\end{array}$ \\
\hline Guerrero & $\begin{array}{l}\text { 1. Ambiente y sociedad; 2. Patrimonio natural del Estado de Guerrero; } 3 . \\
\text { Problemas con fuerte impacto ambiental en el estado de Guerrero; } 4 \text {. Legislación } \\
\text { ambiental en el estado de Guerrero; } 5 \text {. Acciones para la sustentabilidad en las } \\
\text { regiones de Guerrero. }\end{array}$ \\
\hline
\end{tabular}




\begin{tabular}{|c|c|}
\hline Hidalgo & $\begin{array}{l}\text { 1. Calentamiento global y cambio climático; } 2 \text {. Residuos sólidos, producción y } \\
\text { consumo sustentable; } 3 \text {. Manejo democrático y sustentable del agua; } 4 \text {. } \\
\text { Racionalidad, sociedad y biodiversidad; } 5 \text {. Sustentabilidad, democracia y derechos } \\
\text { humanos. }\end{array}$ \\
\hline Jalisco & $\begin{array}{l}\text { 1. Los adolescentes: calidad de vida y sustentabilidad; } 2 \text {. La organización de la } \\
\text { vida: cuencas en la entidad; } 3 \text {. Diversidad, cultura y patrimonio natural; } 4 \text {. La huella } \\
\text { ecológica en la organización de la vida; } 5 \text {. Huella ecológica y acciones para el } \\
\text { futuro. }\end{array}$ \\
\hline Michoacán & $\begin{array}{l}\text { 1. Los jóvenes y la riqueza natural de Michoacán; 2. Relación ser humano- } \\
\text { naturaleza; 3. El agua y las áreas naturales protegidas del estado; } 4 \text {. } \\
\text { Sustentabilidad: un desarrollo diferente; } 5 \text {. Proyectos ambientales para impulsar la } \\
\text { sustentabilidad. }\end{array}$ \\
\hline Morelos & $\begin{array}{l}\text { 1. Panorama de la crisis ambiental y aportes metodológicos para confrontarlos; } 2 \text {. } \\
\text { Residuos sólidos urbanos o basura: un problema por resolver; } 3 \text {. Agua; } 4 \text {. Cambio } \\
\text { climático; } 5 \text {. Suelo. }\end{array}$ \\
\hline Querétaro & $\begin{array}{l}\text { 1. Impacto humano en el ambiente; } 2 \text {. El uso del agua; } 3 \text {. El agua, un problema de } \\
\text { escasez en Querétaro; } 4 \text {. El manejo adecuado de los residuos sólidos; } 5 \text {. La } \\
\text { biodiversidad en Querétaro. }\end{array}$ \\
\hline $\begin{array}{l}\text { Quintana } \\
\text { Roo }\end{array}$ & $\begin{array}{l}\text { 1. Conocimiento del ambiente en Quintana Roo; 2. Valor en la biodiversidad; } 3 . \\
\text { Problemas ambientales del estado de Quintana Roo; Programa para la } \\
\text { sustentabilidad en Quintana Roo; 5. Problemas ambientales: acciones locales, } \\
\text { impactos globales. }\end{array}$ \\
\hline Tabasco & $\begin{array}{l}\text { stentable; 2. Manejo y calidad de los recursos hídricos; } 3 \text {. Residuos } \\
; \text {;. Agricultura sustentable; } 5 \text {. Compromiso con la comunidad. }\end{array}$ \\
\hline Tamaulipas & $\begin{array}{l}\text { 1. Biodiversidad en Tamaulipas; } 2 \text {. Importancia de las regiones naturales en } \\
\text { Tamaulipas; } 3 \text {. La contaminación causada por la industria y la sociedad; } 4 \text {. } \\
\text { Protección ambiental para el desarrollo sustentable; } 5 \text {. Participación social en la } \\
\text { conservación del ambiente. }\end{array}$ \\
\hline Tlaxcala & $\begin{array}{l}\text { 1. El ambiente en mi comunidad y Estado; } 2 \text {. La cultura ambiental y mi contexto; } 3 . \\
\text { Problemática ambiental en Tlaxcala; 4. Participación ciudadana para la } \\
\text { sustentabilidad; } 5 \text {. Hacia un desarrollo sustentable en Tlaxcala. }\end{array}$ \\
\hline Veracruz & $\begin{array}{l}\text { 1. Sistemas ambientales en un mundo complejo y cambiante; } 2 \text {. Proyectos } \\
\text { ambientales en la comunidad; } 3 \text {. Problemas ambientales en la comunidad; } 4 \text {. } \\
\text { Alternativas para la atención ambiental; } 5 \text {. Desarrollo sustentable y calidad de vida. }\end{array}$ \\
\hline Zacat & $\begin{array}{l}\text { 1. Relación del ser humano y su ambiente; } 2 \text {. La riqueza natural de Zacatecas; } 3 . \\
\text { La biodiversidad de Zacatecas; } 4 \text {. Crisis ambiental; } 5 \text {. Desarrollo sustentable. }\end{array}$ \\
\hline
\end{tabular}

Fuente: SEP, 2013a.

En los programas es posible observar que los contenidos no se refieren solo a los aspectos de la ecología, se han incluido temas relacionados con la cultura y la sociedad. La visión de los programas respecto a los problemas ambientales es amplia, en comparación con los primeros programas de educación ambiental, aunque enfatizan cuestiones particulares de cada Estado no se dejan fuera cuestiones globales, como el cambio climático global.

Los programas están estructurados en cinco bloques. En el $14.73 \%$ de los títulos de los bloques se usa el concepto de desarrollo sustentable; en un $14.73 \%$ se hace referencia al concepto de sustentabilidad; en un $12.63 \%$ se alude a los distintos aspectos del agua; en un 
$10.52 \%$ se hace mención de los componentes de la naturaleza; y un $6.25 \%$ respecto a los residuos sólidos. Se hace evidente el enfoque de la educación ambiental para la sustentabilidad.

Asimismo, es evidente que no se tocan aspectos relevantes para la formación ambiental de los adolescentes, quedan fuera temas como la pobreza y marginación social, historia del capitalismo, el modelo económico hegemónico actual, el antropocentrismo, el ecocentrismo, los pueblos originarios y la diversidad cultural, la alienación y la búsqueda de sentido y la conciencia planetaria, entre otros. Temas que pueden hacer posible fomentar en los estudiantes los valores ambientales que los conduzcan a comportamiento responsable con el medio ambiente, fundamentados en una ética ambiental.

\section{Implicaciones para la docencia}

A partir de la difusión del desarrollo sustentable o sostenible a nivel internacional del Informe Brundtland titulado"Nuestro Futuro Común" en 1987, inició un proceso de replanteamiento en América Latina del concepto de sustentabilidad. Existen diversas formas de entender a la sustentabilidad, como se observa en la tabla 6.

Tabla 6. Aspectos de la sustentabilidad

\begin{tabular}{|l|l|l|l|}
\hline \multicolumn{1}{|c|}{ Elementos } & Sustentabilidad débil & Sustentabilidad fuerte & $\begin{array}{l}\text { Sustentabilidad súper- } \\
\text { fuerte }\end{array}$ \\
\hline Perspectiva & Antropocéntrica & Antropocéntrica & Biocéntrica \\
\hline Desarrollo & Crecimiento material & Crecimiento material & $\begin{array}{l}\text { Calidad de vida, calidad } \\
\text { ecológica }\end{array}$ \\
\hline Naturaleza & Capital natural & Capital natural & Patrimonio natural \\
\hline Valoración & Instrumental & Instrumental ecológica & Múltiple, intrínseca \\
\hline Actores & Consumidores & Consumidor ciudadano & Ciudadano \\
\hline Escenario & Mercado & Sociedad & Sociedad \\
\hline Saber científico & $\begin{array}{l}\text { Conocimiento } \\
\text { privilegiado }\end{array}$ & $\begin{array}{l}\text { Conocimiento } \\
\text { privilegiado }\end{array}$ & $\begin{array}{l}\text { luralidad } \\
\text { conocimiento }\end{array}$ \\
\hline Otros saberes & Ignorados & Minimizados & $\begin{array}{l}\text { Respetados, } \\
\text { incorporados }\end{array}$ \\
\hline Prácticas & Gestión técnica & $\begin{array}{l}\text { Gestión } \\
\text { consultiva }\end{array}$ & técnical Política ambiental \\
\hline Justicia social & Improbable & Posible & Necesaria \\
\hline Justicia ecológica & Imposible & Posible & \\
\hline
\end{tabular}

Fuente: Eduardo Gudynas, 2009.

En los programas de estudio analizados se encuentran elementos de la sustentabilidad débil y fuerte. Estos elementos nos indican el predominio de representaciones 
antropocéntricas. En estas representaciones, el medio ambiente es observado en función de los beneficios que aportan a las distintas actividades humanas.

Los contenidos de los programas privilegian la idea de las actividades en beneficio la naturaleza, que no refleja realmente una conservación real de la naturaleza, sino para continuar con la explotación de los recursos que ella provee.

La sustentabilidad y la economía, en su interacción mutua, representan un desplazamiento filosófico hacia la comprensión de la Tierra como un recurso más y representa la aplicación de proyecciones de largo plazo en el uso de los recursos (Irwin, 2008). Esta visión prevalece en los contenidos de los programas analizados.

La sustentabilidad débil se asocia más a las directrices del desarrollo sustentable que en las propuestas de la educación ambiental, esto, en el sentido que propone aprovechar los recursos no sólo económicos sino también los naturales con la intención de producir los satisfactores que la sociedad demanda. También resalta la idea de cuidar los ecosistemas mediante la administración eficiente de los recursos.

Existe un menor número de elementos asociados a la sustentabilidad fuerte, en éstas, se considera a la sustentabilidad como algo que llegó a tiempo para atender la problemática ambiental, de manera intervencionista e instrumentalista.

La sustentabilidad fuerte se asocia con la educación ambiental para la sustentabilidad ya que incorpora en sus acciones, condiciones para que la vida perdure, se consideran aspectos ambientales, sociales y políticos. En esta perspectiva se incorporan contenidos referidos al equilibrio real entre las especies vivas y la utilización de los recursos. Se considera la satisfacción de las necesidades futuras de los otros con miras al desarrollo económico y se enfatiza el cuidado el medio ambiente al llevar a cabo cualquier actividad.

La sustentabilidad súper-fuerte se vincula con la educación popular ambiental esta se considera como una propuesta transformadora que aporta elementos para un cambio de creencias, actitudes, hábitos, conocimientos y comportamientos que impacten de una manera positiva en el ambiente, favoreciendo una relación más amigable del ser humano con el ambiente.

Este análisis devela que hacen falta contenidos de la sustentabilidad súper-fuerte en los programas de educación ambiental. En el nivel medio básico es importante generar acciones para que en los programas de estudio se transite de la sustentabilidad débil, hacia la sustentabilidad fuerte y la sustentabilidad súper-fuerte.

La principal limitante para el desarrollo de los programas en educación ambiental en 
las escuelas secundarias, se relaciona con la formación en este campo de los docentes, ya que por lo general, predomina en ellos una representación naturalista del medio ambiente y una representación ecologista de la educación ambiental. Algunos profesores consideran a la educación ambiental como ecología, vinculada a los problemas ambientales, Molfi-Goya (2000).

Por otra parte los profesores tienen confusiones en diferenciar a la sustentabilidad, del desarrollo sustentable. Los usan como sinónimos, vinculados a las actividades productivas, como "economía verde.," o "bonos verdes." En un estudio previo que utilizó cartas asociativas para identificar las representaciones sociales (RS) de los profesores, se encontró que por lo general dejan fueran términos asociados a la sustentabilidad fuerte y súper-fuerte; ya que no forman parte de sus representaciones.

Las RS naturalistas del medio ambiente tienen implicaciones importantes en la educación ambiental, ya que son la base de una educación ambiental naturalista, recursista/conservacionista, en la que se privilegia el cuidado de la naturaleza. Ubican al ser humano como ajeno a ésta, además tiene el papel de protegerla y conservarla... (Calixto, 2013, p. 16)

Por otra parte pocos profesores conocen las implicaciones de la sustentabilidad para la docencia; esta situación se propicia en gran medida, cuando en los programas de estudio prevalecen contenidos referidos a la sustentabilidad débil y la sustentabilidad fuerte. Los profesores han de comprender a la sustentabilidad como una alternativa de formación de conciencias ambientalmente responsables.

Una de las principales recomendaciones que los mismos programas incluyen, es que los profesores se apropien de los aprendizajes de la asignatura, los cuales no se restringen a los elementos naturales y adquieran a su vez, las competencias necesarias para desarrollan el enfoque sustentable de la educación ambiental. Sin embargo en la oferta de cursos de formación continua relacionados con la educación ambiental, para profesores de educación secundaria y en general para los profesores de todos los niveles es muy reducida. En los cursos y diplomados que se ofrecen con cobertura estatal, y en los diplomados con cobertura nacional a los profesores en servicio de escuelas secundarias, para el ciclo escolar 20122013, no existen propuestas relacionadas con la educación ambiental. Sólo se ofrecen cuatro cursos de cubertura nacional, como se observa en la tabla 7. 
Tabla 7. Cursos de formación continua (2012-2013)

\begin{tabular}{|c|c|c|}
\hline Curso & Institución responsable & $\begin{array}{l}\text { Dirigido a profesores de } \\
\text { niveles y modalidades }\end{array}$ \\
\hline $\begin{array}{l}\text { Cambio climático: } \\
\text { evidencia y acción }\end{array}$ & $\begin{array}{l}\text { Centro de capacitación y } \\
\text { educación para el desarrollo } \\
\text { sustentable, SEMARNAT-SEP }\end{array}$ & $\begin{array}{l}\text { ylnicial, preescolar, primaria } \\
\text { olregular e indígena), secundaria, } \\
\text { telesecundaria, educación física, } \\
\text { especial, extraescolar y artística } \\
\text { CAPEP }\end{array}$ \\
\hline $\begin{array}{l}\text { Prácticas educativas para } \\
\text { desarrollo sustentable }\end{array}$ & $\begin{array}{l}\text { Universidad Tecnológical } \\
\text { Emiliano Zapata del Estado de } \\
\text { Morelos }\end{array}$ & $\begin{array}{l}\text { alnicial, preescolar, primaria } \\
\text { e(regular e indígena), secundaria, } \\
\text { telesecundaria, educación física, } \\
\text { especial, extraescolar y artística }\end{array}$ \\
\hline $\begin{array}{l}\text { Que cambia } \\
\text { climático }\end{array}$ & $\begin{array}{l}\text { Dirección General de Formación } \\
\text { Continua de Maestros en } \\
\text { Servicio }\end{array}$ & $\begin{array}{l}\text { nlnicial, preescolar, primaria } \\
\text { n(regular e indígena), secundaria, } \\
\text { telesecundaria, educación física, } \\
\text { especial, extraescolar y artística } \\
\text { CAPEP }\end{array}$ \\
\hline $\begin{array}{l}\text { Economía, Ambiente y Sociedad, } \\
\text { ejes de la Sustentabilidad: un } \\
\text { enfoque para la enseñanza } \\
\text { básica }\end{array}$ & Universidad Tecnológica de Tula- & -Secundaria \\
\hline
\end{tabular}

Fuente: SEPb, 2013.

Es necesario consolidar la oferta de cursos referidos a la educación ambiental, para que los docentes desarrollen una mirada crítica a los problemas ambientales, se requiere que los profesores acepten:

...el desafío de abordar el conflicto en los temas ambientales, implica que el docente sea capaz de "soportar" las preguntas de sus alumnos. Pensar la realidad les permite desarrollar su juicio crítico y fundamentado, que será particularmente necesario y útil en aquéllos que se inserten en la sociedad desde roles activos y comprometidos con el cuidado y la preservación del ambiente local, nacional o global... (Damin y Monteleone, 2002, pp. 68-69)

En los lineamientos nacionales de la asignatura Estatal se señala que:

A fin de que los maestros estén capacitados para impartir los programas diseñados, las autoridades estatales deberán considerar qué estrategias de actualización requieren, éstas abarcan las acciones realizadas para dar a conocer el programa en cada plantel educativo; el intercambio de experiencias e información sobre avances en su aplicación; las estrategias empleadas para reforzar temas; el uso y aprovechamiento de materiales; las dificultades que se enfrentaron en su aplicación y las formas utilizadas para resolverlas; las 
sugerencias para posteriores acciones de actualización sobre el programa, etcétera (SEPb, 2006, p. 28)

En este sentido, los docentes requieren de apoyos institucionales y de formación y/o actualización en educación ambiental, de tal forma que puedan desarrollar los contenidos de los programas y también incidir en la formación de los valores ambientales.

Una de las implicaciones para la docencia derivada del análisis de los programas corresponde al reconocimiento de los valores ambientales en la sustentabilidad, estos valores se encuentran ausentes en los programas analizados a pesar de que estos valores aportan elementos para preferir determinadas acciones sobre otras. Los valores no se dicen, se viven. Se da testimonio de ellos en gestos ejemplares, vividos y sufridos. Ellos son los que convencen y cautivan. Y, a través de ellos, el pensamiento se deja provocar (Boff,1998, p. 9).

Los valores permiten tomar decisiones respecto a las acciones que pueden tomarse respecto al medio ambiente. En este sentido las asignaturas estatales de educación ambiental, pueden constituir una experiencia exitosa, cuando incorporen aspectos como los valores ambientales.

\section{Reflexiones finales}

El nivel medio básico corresponde a la etapa conclusiva de la educación básica en México; etapa en la que los jóvenes consolidan su formación integral, de acuerdo a los propósitos de este nivel; formación integral que incluye conocimientos, actitudes y valores ambientales. Es por ello necesario reconocer, los problemas, avances y retos de la educación ambiental en este nivel educativo.

Problemas. En los actuales programas de estudio la sustentabilidad no se describe como un proceso, sino como algo prescriptivo, como un conocimiento amplio y solucionador.

La sustentabilidad se presenta como algo ligado a los problemas ambientales y a la preocupación del agotamiento de los recursos naturales; no se vinculación las decisiones tomadas por los gobernantes, relacionadas con la instrumentación y ejecución de la política económica y por consiguiente educativa de un país. La sustentabilidad también se representa como algo propio de las acciones y comportamientos de la sociedad como colectivo, se diluye la responsabilidad de los principales actores responsables de los problemas ambientales. Aspectos que han de ser tomados en cuenta en una educación ambiental para la sustentabilidad; se han de incorporar información, que contribuye a la 
formación y/o consolidación de actitudes y valores ambientales sustentables.

Avances. La declaración de compromisos de diversas instituciones y sectores para hacer realidad los propósitos de la Década de la Educación en Desarrollo Sustentable, la elaboración y el desarrollo de acciones de la Estrategias de Educación Ambiental para la Sustentabilidad en México, la inclusión de la educación ambiental como campo transversal en la Reforma Integral de la Educación Básica y el desarrollo de programas estatales referidos a esta educación, constituyen algunos de los principales avances. La inclusión de la educación ambiental como eje temático en la reforma de la educación básica, y su concreción en el campo formativo de formación exploración y comprensión del mundo natural y social es un acierto. De igual forma el diseño de las asignaturas estatales con objetivos y temas referidos al medio ambiente.

Retos. La educación ambiental para la sustentabilidad ha de orientarse hacia el desarrollo de acciones a largo plazo en el nivel medio básico, con lo cual se propicie una continuidad de los avances obtenidos. Un reto importante, es la incorporación de educadores ambientales en el diseño de programas de estudio, sensibles a los problemas ambientales, en sus distintas dimensiones, congruentes al contexto nacional y regional, sin dejar fuera los aspectos planetarios.

Uno de los principales retos de la educación ambiental es el predominio de los elementos de la sustentabilidad débil, en donde se le concibe como receta, con la prescripción de algunas ideas con sus tiempos y condiciones de uso para mejorar la salud del ambiente o curar de algún malestar al mismo. Esta concepción ha de transformarse, en donde se transite a la sustentabilidad súper-fuerte, y se posibilite el cuestionamiento del origen de los múltiples problemas ambientales.

Para superar los problemas, consolidar los avances y enfrentar los retos, es necesario vincular la educación ambiental con el fomento de valores, con base en el conocimiento del origen y efectos de los diversos problemas ambientales locales, regionales, estatales y globales.

Esto es posible si se logra alfabetizar ambientalmente a los tomadores de decisiones de los distintos niveles del sector educativo, los profesores de los distintos niveles educativos y la atención y seguimiento de los proyectos ambientales generados por los estudiantes de escuelas secundarias.

También se requiere el impulso de la investigación en educación ambiental, la cual propicie la construcción de un andamiaje teórico, con lo cual se adquieran e intercambien 
conocimientos. La conjunción de todos estos aspectos puede ser la base para que los programas de educación ambiental se constituyan en una experiencia exitosa en las escuelas secundarias.

\section{Agradecimientos}

Agradezco el apoyo recibido del Área académica 2. Diversidad e Interculturalidad en la elaboración del presente ensayo.

\section{Referencias}

Boff, Leonardo. (1998). Brasas y bajo las cenizas (2a. ed.). Barcelona: Trotta.

Calixto, Raúl. (2013). Educación ambiental en representaciones de docentes de escuelas secundarias. CPU-e Revista de Investigación Educativa 16, Veracruz, México: Universidad Veracruzana. Recuperado de http://www.redalyc.org/articulo.oa?id=283128328003

Comisión Bruntland. (1987). Nuestro Futuro Común. New York: Oxford University Press.

Damin, Roberto y Monteleone, Adrián. (2002). Temas ambientales en el aula. Una mirada crítica desde las ciencias sociales. Buenos Aires: Paidós.

González-Gaudiano, Edgar. (1997). Educación ambiental. Historia y conceptos a veinte años de Tbilisi. México: Sistemas Técnicos de Edición.

Gudynas, Eduardo. (2009). Desarrollo sostenible: posturas contemporáneas y desafíos en la construcción del espacio urbano. Vivienda Popular, (18), 12-19. Montevideo: Facultad de Arquitectura.

Gutiérrez, José y Priotto, Guillermo. (2008). Sobre un modelo latinoamericano de desarrollo curricular descentralizado en Educación Ambiental para la Sustentabilidad. Revista Mexicana de Investigación Educativa, 13(37), 529-571.

Irwin, Ruth. (2008). Posneoliberalismo: de la educación ambiental a la educación para la sustentabilidad. En Edgar González Gaudiano (Coord.), Educación, medio ambiente y sustentabilidad (pp. 176-212), México: Siglo XXI, Universidad Autónoma de Nuevo León.

Krippendorff, Klaus. (1990). Metodología del análisis de contenido. Teoría y Práctica. Barcelona: Paidós Ibérica.

Martínez, Róger. (2007). Aspectos políticos de la educación ambiental. Actualidades Investigativas en Educación, 78(3), 1-25. Recuperado de http://revista.inie.ucr.ac.cr/index.php/aie/article/view/192 
Molfi-Goya, Eneida. (2000). Deconstrucción de las representaciones sobre el medio ambiente y la educación ambiental, Tópicos en Educación ambiental, vol.2, No. 4. México: Universidad Nacional Autónoma de México/Secretaria del Medio Ambiente, Recursos Naturales y Pesca, 33-40.

Piñuel, José Luis y Gaitán Juan Antonio. (1995). Metodología general. Conocimiento científico e investigación en la comunicación social. Madrid: Síntesis.

Porta, Luiz y Silva Miriam. (2003). La investigación cualitativa. El análisis de contenidos en la investigación educativa. Recuperado de http://www.uccor.edu.ar/paginas/REDUC/porta.pdf

Secretaría de Educación Pública, México. (2004). Acuerdo 348. Diario Oficial de la Federación, México: Secretaría de Gobernación.

Secretaría de Educación Pública, México. (2006a). Acuerdo 384. Diario Oficial de la Federación, México: Secretaría de Gobernación.

Secretaría de Educación Pública, México. (2006b). Educación básica. Secundaria. Asignatura Estatal. Lineamientos Nacionales. México: SEP.

Secretaría de Educación Pública, México. (2010). Acuerdo 540. Diario Oficial de la Federación. México: Secretaría de Gobernación.

Secretaría de Educación Pública, México. (2011a). Plan de estudios 2011. Educación básica. Recuperado de http://basica.sep.gob.mx/dgdc/sitio/pdf/PlanEdu2011.pdf

Secretaría de Educación Pública, México. (2011b). Acuerdo sectorial número 592. Recuperado http://dof.gob.mx/nota detalle.php?codigo=5205518\&fecha=19/08/2011

Secretaría de Educación Pública, México. (2013a). Catálogo Nacional 2013. Programas de estudio de Asignatura Estatal. Plan de estudios 2011. (Versión digital, PDF). Recuperado de http://www.curriculobasica.sep.gob.mx/index.php/estatal/catalogo$\underline{\text { nacional }}$

Secretaria de Educación Pública, México. (2013b). Oferta de cursos de formación continua a nivel nacional. Recuperado de http://cecmslp.seslp.gob.mx/CCN.pdf

Secretaría del Medio Ambiente y Recursos Naturales, México (2006). Estrategia de Educación Ambiental para la Sustentabilidad en México. México: Semarnat. 